\title{
Adaptive Correction Algorithm for OFDM-IDMA Systems with Carrier Frequency OFFSET in a Fast Fading Multipath Channel
}

\author{
Muyiwa B. Balogun ${ }^{1}$, Olutayo O. Oyerinde ${ }^{2}$ and Stanley H. Mneney ${ }^{1}$ \\ ${ }^{1}$ School of Engineering, Discipline of Electrical, Electronic and Computer \\ Engineering, University of KwaZulu-Natal, Durban, 4041, South Africa, Email: \\ 212561614@stu.ukzn.ac.za; Mneneys@ukzn.ac.za \\ ${ }^{2}$ School of Electrical and Information Engineering, University of the \\ Witwatersrand, Johannesburg, 2050, South Africa, Olutayo.Oyerinde@wits.ac.za
}

Received 27 October 2013; Accepted 5 December 2013;

Publication 23 January 2014

\begin{abstract}
The Orthogonal Frequency Division Multiplexing-Interleave Division Multiple Access (OFDM-IDMA) scheme, which offers significant improvement on the performance of the conventional IDMA technique, has been in the forefront of recent mobile communication researches as it is expected to deliver a high quality, flexible and efficient high data-rate mobile transmission. Most papers on OFDM-IDMA scheme assume a system free of carrier frequency offset. However, the scheme is susceptible to synchronization errors and performance degradation because of the presence of OFDM, which is highly sensitive to carrier frequency offset (CFO) especially at the uplink. The effect of CFO on the performance of the scheme, in a slow fading multipath channel scenario, is therefore investigated, and analyzed. Also, the effect of CFO on the performance of the OFDM-IDMA scheme, in a fast fading multipath channel, which has not been hitherto reported in literature, is investigated and analyzed. An LMS-based adaptive synchronization algorithm is therefore employed to mitigate the degrading impact of carrier frequency offset errors on the OFDMIDMA scheme. Simulation results clearly show that the presence of CFO degrades the performance of the system and that performance degradation
\end{abstract}

Journal of Cyber Security, Vol. 2 No. 3 \& 4, 201-220.

doi: $10.13052 / \mathrm{jcsm} 2245-1439.231$

(C) 2014 River Publishers. All rights reserved. 
due to CFO, increases in a fast fading multipath channel in comparison with slow fading channel scenario. Furthermore, results show substantial improvement in the overall output of the system upon the application of the adaptive synchronization algorithm, which is implemented in both slow, and fast fading Rayleigh multipath channel scenarios.

Keywords: OFDM, IDMA, OFDM-IDMA, CFO, LMS.

\section{Introduction}

The need for better quality of service, improved capacity and high data-rate transmission, which has become nonnegotiable, have informed the continuous search for a reliable and efficient multicarrier scheme. The Code Division Multiple Access (CDMA) and the Orthogonal Frequency Division Multiplexing (OFDM) techniques rank high above other multiuser schemes due to their inherent advantages. The OFDM technique has particularly become difficult to ignore and almost indispensible because of its support for high data rate transmission and the ability to suppress ISI without much difficulty. Thus, OFDM has now become the bedrock of most recent multicarrier schemes in wireless communications. The combination of the CDMA and the OFDM technique to form a hybrid scheme of OFDM-CDMA has gained prominence and considered attractive due to the diversity and radio resource management flexibility offered. As studied in [1, 2, 3], there are various methods of combining the OFDM and the CDMA schemes, but the main idea behind the multicarrier CDMA hybrid scheme is to perform a spreading operation on transmitted signals which are then converted into parallel streams. The serial-to-parallel converted data are then modulated over different subcarriers, which are mutually orthogonal, and transmitted over the radio channel. The spreading code assigned to each user is to enable signal separation at the receiver. However, due to diverse level of fading and attenuation experienced by the transmitted signals, orthogonality is lost among subcarriers. This leads to Multiple Access Interference (MAI), causing high degradation in cellular performance, which becomes severe as the number of simultaneous users increases.

In an effort to address the MAI in multicarrier CDMA (MC-CDMA), the Multiuser Detection (MUD) technique was introduced. The priority of the MUD is to subtract interfering signals from the input signal of each user in the system. However, the MUD technique utilized in MC-CDMA comes with 
associated complexities and high cost [4, 5, 6]. Various MUD techniques have been proposed to address the high complexity of the MUD technique, but with little success. The complexity of the MUD tends to increase exponentially as the number of active subscribers increases. Recent studies have explored the possibility of the artificial neural network for multiuser detection [7] but these techniques tend to compromise system performance and efficiency for reduced complexity. The MUD challenge in MC-CDMA therefore remains and there has been a continuous search for an efficient and reliable scheme with low complexity.

To this end, a new multiuser scheme was recently proposed by Li Ping called the Interleave Division Multiple Access (IDMA) [8]. This scheme employs a simple low cost chip-by-chip iterative method for its multiuser detection. The IDMA scheme, which offers a lower system complexity compared to MC-CDMA [9], relies solely on interleaving as the only means of identifying signals from active users in the system. In a bid to achieve an improved cellular performance of the IDMA over multipath, Mahafeno in 2006 proposed an OFDM-based hybrid scheme called the OFDM-IDMA scheme [10]. The newly proposed multicarrier IDMA (MC-IDMA) scheme therefore combats ISI and MAI effectively over multipath with low complexity. The multicarrier scheme ensures a better cellular performance, high diversity order, and spectral efficiency compared to the MC-CDMA scheme. Thus, the scheme combines all the inherent advantages of the conventional IDMA and the OFDM technique. The associated MUD is of low cost and low complexity per user, which is independent of the number of simultaneous users in the system $[11,12]$.

Major studies on the OFDM-IDMA scheme focus only on the implementation of the scheme in a perfect scenario, assuming that there are no synchronization errors in the system. This is not obtainable in practice. The scheme becomes susceptible to synchronization errors because of the presence of the OFDM technique, which is highly sensitive to carrier frequency offsets (CFO) especially at the uplink, where different users are transmitting asynchronously, experiencing different levels of channel fading and delays. The CFO is caused mainly by Doppler Shifts or because of instabilities in the local oscillators [13]. CFO causes Inter-channel Interference (ICI) and loss of orthogonality among the sub-carriers, which in turn leads to performance degradation.

This paper, therefore, investigates and analyzes the impact of CFO on the general performance of the OFDM-IDMA scheme. Also, the performance of the system in a fast fading multipath channel is investigated and as well 
analyzed. As an extension of the performance analysis of the OFDM-IDMA scheme with synchronization errors, carried out in [14], an LMS-based adaptive synchronization algorithm is then introduced to combat the degrading impact of synchronization errors on the OFDM-IDMA system. Simulation results show that the performance of the system degrades as the CFO increases and the system also experiences degradation as the velocity increases in the fast fading multipath channel. An improved system output is however obtained upon the application of the LMS-based adaptive synchronization algorithm.

The rest of the paper is organized as follows: Section II describes the system model of the OFDM-IDMA scheme in the presence of CFO. Section III describes the system performance in the presence of CFO. Section IV presents the LMS-based adaptive synchronization algorithm. Section V discuses simulation results and finally Section VI gives the conclusion with a summary of the major results.

\section{System Model}

The OFDM technique $[15,16]$, which involves the splitting of high-rate data streams into a large number of lower-rate data streams, which are transmitted, while maintaining orthogonality, across multiple narrowband sub-carriers, solves the problem of inter-symbol Interference (ISI), which is encountered while transmitting high-rate data streams across multipath channels. As long as orthogonality is maintained, there will be no interference between sub-carriers and this will enable the receiver to separate signals carried by each sub-carrier. Unlike the conventional Frequency Division Multiplexing (FDM) scheme, the spectra of the different modulated sub-carriers overlap in OFDM as seen in Fig. 1(b). This makes OFDM an appropriate scheme for optimum and efficient use of valuable spectrum. Fig. 2 illustrates in block diagram the adaptation of the IEEE standard 802.11a [17] for a baseband OFDM transceiver.

The IDMA technique is a type of multi-user technique where interleavers serve as the sole means of user separation. The interleavers, which are randomly generated, must be different for each user. Adjacent coded data, called "chips," are uncorrelated in this scheme as the interleavers separate the coded sequences, ensuring the simple implementation of the low-cost chip-by-chip detection algorithm [18, 19]. The OFDM transceiver structure in Fig. 2 is incorporated into the IDMA transceiver as shown in fig. 3. Considering a multicarrier IDMA system with $\mathrm{K}$ users transmitting simultaneously, for anyone of the users denoted by $\mathrm{k}$, the input data array is first encoded using a Forward Error Correlation (FEC) code [20]. The coded sequence obtained 
is then interleaved by a randomly generated interleaver to give $x_{k}(n)$. The resulting signal is transmitted over the channel to the receiver. The received signal can be expressed as

$$
\begin{gathered}
r(n)=\sum_{k=1}^{k} x_{k}(n) h_{k}(n)+q(n) \\
=x_{k}(n) h_{k}(n)+\zeta_{k}(n) \\
\zeta_{k}(n)=\sum_{k^{\prime} \neq k} x_{k^{\prime}}(n) h_{k^{\prime}}(n)+q(n)
\end{gathered}
$$

where $h_{k}(\mathrm{n})$, which is assumed to be known at the receiver, is the fading channel coefficient for user $\mathrm{k}, q(n)$ is the additive white Gaussian noise and the multi-user interference with respect to user $\mathrm{k}$ is denoted by $\zeta_{\mathrm{k}}(\mathrm{n})$.

The receiver structure consists of the Elementary Signal Estimator (ESE) and a posterior probability (APP) decoders [20] for each user $k$. The mode of operation of the ESE and the decoders (DECs) is iterative [20] [21], deinterleaving and interleaving process also occurs iteratively between them. The ESE carries out a coarse chip-by-chip detection to roughly subtract the interference among the concurrent users in the multicarrier IDMA system. The chip-by-chip detection is of a very low computational cost and complexity [22]. The outputs of the ESE are the estimated probabilities of the transmitted signals, which are organized sequentially according to the simultaneous users and are fed to the APP decoders [22]. The mode of operation of the ESE and DECs is iterative, where extrinsic information is processed in a turbo-like mode between them. At the last iteration, the APP decoders give the hard decisions based on the refined estimations by the ESE and the logarithm likelihood ratio (LLR) estimate of the ESE and the APP decoders is obtained as [23]

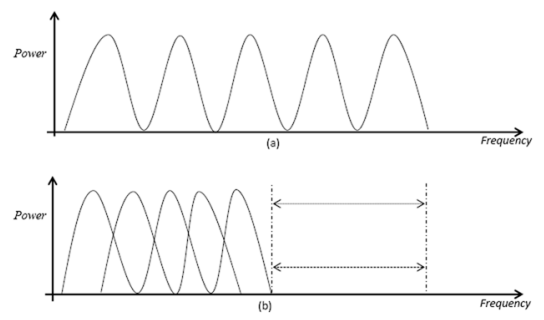

Figure 1 Illustration of the spectrum-saving concept of the OFDM technique (b) compared with the regular FDM scheme (a) 


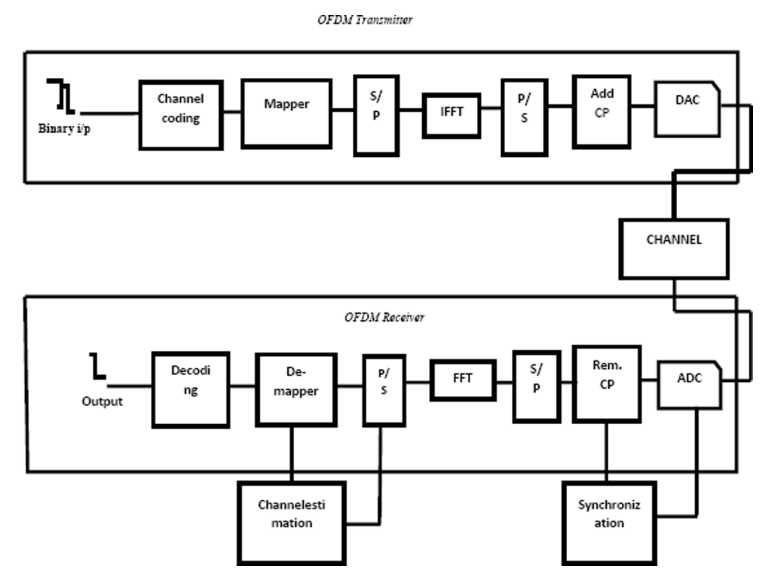

Figure 2 Block diagram of a typical OFDM transceiver

$$
p\left(x_{k}(n)\right) \equiv \log \left[\frac{P_{b}\left(x_{k}(n)=+1\right)}{P_{b}\left(x_{k}(n)=-1\right)}\right]
$$

The outputs of both the ESE and the DECs above are probabilities of the values of the transmitted radio signals at the receiver [20], which will henceforth be stated as $\mathrm{P}_{\mathrm{ESE}}\left(\mathrm{x}_{k}(\mathrm{n})\right.$ and $\mathrm{P}_{\mathrm{DEC}}\left(\mathrm{x}_{k}(\mathrm{n})\right)$ depending on whether they are emanating from the ESE or the APP decoders at the receiver. Therefore, considering the fading channel represented by the coefficient $h$, the ESE employs the received signal $r(n)$ and the LLR for its operation, so that the resulting output is obtained as [23]

$$
\begin{gathered}
\log \left[\frac{P_{b}\left(x_{k}(n)=+1 \mid r(n), h\right)}{P_{b}\left(x_{k}(n)=-1 \mid r(n), h\right)}\right]=\log \left[\frac{P_{b}\left(x_{k}(n)=+1\right), h}{P_{b}\left(x_{k}(n)=-1\right), h}\right] \\
+p_{E S E}\left(x_{k}(n)\right),
\end{gathered}
$$

where the first part of the equation can be expressed as

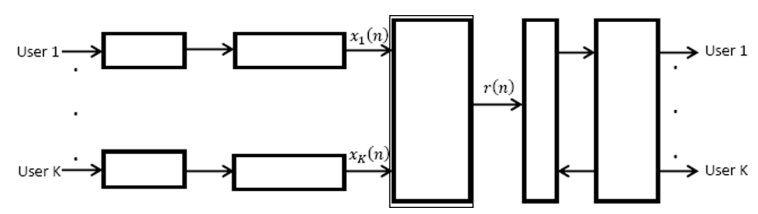

Figure 3 Block diagram of an OFDM-IDMA transceiver 


$$
e_{E S E}\left(x_{k}(n)\right)=\log \left[\frac{P_{b}\left(x_{k}(n)=+1\right), h}{P_{b}\left(x_{k}(n)=-1\right), h}\right],
$$

which is the extrinsic LLR about the transmitted signal $x_{k}(\mathrm{n})$ based on the characteristics of the fading channel and the a priori information of concurrent users in the system [23].

Considering the ESE chip-by-chip detection in a quasi-static multipath channel where the BPSK signaling is used and the transmitted signal $x_{k}(n)$ is treated as a random variable, $e_{E S E}\left(x_{k}(\mathrm{n})\right.$ is used to coarsely update the $a$ priori LLR $p\left(x_{k}(n)\right)$, which is obtained from (4) as [23]

$$
E\left(x_{k}(n)\right)=\left[\frac{\left.\exp \left(p_{E S E}\left(x_{k}(n)\right)\right)-1\right)}{\left.\exp \left(p_{E S E}\left(x_{k}(n)\right)\right)+1\right)}\right]=\tanh \left(p_{E S E}\left(x_{k}(n)\right) / 2\right),
$$

$$
\operatorname{Var}\left(x_{k}(n)\right)=1-\left(E\left(x_{k}(n)\right)\right)^{2},
$$

where $\mathrm{E}\left(\mathrm{x}_{k}(\mathrm{n})\right)$ and $\operatorname{Var}\left(x_{k}(\mathrm{n})\right)$ are the mean and variance of the transmitted signal $x_{k}(\mathrm{n})$ respectively. Using the central limit theorem, the interference $\zeta_{k}(\mathrm{n})$ in (3) can be estimated by a zero-mean Gaussian variable with variance $\sigma^{2}$, given as [23]

$$
\begin{gathered}
E\left(\zeta_{k}(n)\right)=\sum_{k^{\prime} \neq k}^{K} h_{k^{\prime}}(n) E\left(x_{k^{\prime}}(n)\right), \\
\operatorname{Var}\left(\zeta_{k}(n)\right)=\sum_{k^{\prime} \neq k}^{K}\left|h_{k^{\prime}}(n)\right|^{2} \operatorname{Var}\left(x_{k^{\prime}}(n)\right)+\sigma^{2} .
\end{gathered}
$$

Applying the Gaussian estimation to the received signal in (2), the output of the ESE in (3) is obtained as [23]

$$
\begin{aligned}
& e_{E S E}\left(x_{k}(n)\right)=2 h_{k}(n) \cdot \frac{r(n)-E\left(\zeta_{k}(n)\right)}{\operatorname{Var}\left(\zeta_{k}(n)\right)} \\
& =2 h_{k}(n) \cdot \frac{r(n)-E(r(n))+h_{k}(n) E\left(x_{k}(n)\right)}{\operatorname{Var}(r(n))-\left|h_{k}(n)\right|^{2} \cdot \operatorname{Var}\left(x_{k}(n)\right)} .
\end{aligned}
$$

The estimated mean and variance of the received signal based on (1) is therefore obtained as [23]

$$
E(r(n))=\sum_{k^{\prime} \neq 1}^{K} h_{k^{\prime}}(n) E\left(x_{k^{\prime}}(n)\right),
$$




$$
\operatorname{Var}(r(n))=\sum_{k^{\prime} \neq 1}^{K}\left|h_{k^{\prime}}(n)\right|^{2} \operatorname{Var}\left(x_{k^{\prime}}(n)\right)+\sigma^{2} .
$$

In $[25,26]$, various methods for carrier frequency offset estimation have been presented and these methods of CFO estimation can be put to use in the OFDM-IDMA system. Therefore, the carrier frequency offset is assumed to be known for users in this OFDM-IDMA model.

\section{System Performance Analysis}

The expression in ( $(1)$ represents only a perfect scenario where the CFO is zero. Considering, therefore, the effect of CFO on the received baseband signal, (1) can be rewritten as

$$
r_{c}(n)=\sum_{k=1}^{K} x_{k}(n) h_{k}(n) e^{j 2 \pi \varepsilon_{k} n / N}+q(n),
$$

where $\mathrm{n}$ represents the sub-carrier index, $\mathrm{N}$ is the number of sub-carriers and $\epsilon_{k}, \epsilon_{k} \ll 0.5$ [24], represents the normalized CFO. After DFT processing in the presence of CFO, equation (14) becomes:

$$
\begin{gathered}
R_{c}(m)=\sum_{n=0}^{N-1} r_{c}(n) e^{-j 2 \pi \varepsilon_{k} m / N} \\
=X_{k}(m) H_{k}(m)+\sum_{k^{\prime} \neq k} X_{k^{\prime}}(m) H_{k^{\prime}}(m)+\mu_{k}(m)+Q(m)
\end{gathered}
$$

The presence of CFO in the OFDM-IDMA system and its impact on the performance of the system can be inferred from (14)-(16), which are the modifications of (1)-(3). The second part of (16), which gives the combined interference due to the introduced CFO and the multiuser interference, can be represented by $\zeta_{k}^{\prime}(\mathrm{m})$ given as:

$$
\zeta_{\mathrm{k}}^{\prime}(\mathrm{m})=\sum_{\mathrm{k}^{\prime} \neq \mathrm{k}} \mathrm{X}_{\mathrm{k}^{\prime}}(\mathrm{m}) \mathrm{H}_{\mathrm{k}^{\prime}}(\mathrm{m})+\mu_{\mathrm{k}}(\mathrm{m})+\mathrm{Q}(\mathrm{m})
$$

where $\mathrm{Q}(\mathrm{m})$ represents a Gaussian random variable which can be expressed as [27]: 


$$
Q(m)=\sum_{n=0}^{N-1} q(n) e^{-j 2 \pi n\left(m-\varepsilon_{k}\right) / N},
$$

and $\mu_{k}(m)$ is the interference due to CFO between user $k$ given as $\epsilon_{k}$ and other user $k^{\prime}$ given as $\epsilon_{k^{\prime}}$. This can be expressed following [28] as:

$$
\mu_{\mathrm{k}}(\mathrm{m})=\sum_{\mathrm{n}=0}^{\mathrm{N}-1} \mathrm{e}^{\mathrm{j} 2 \pi \mathrm{n}\left(\varepsilon_{\mathrm{k}^{\prime}}-\varepsilon_{\mathrm{k}}\right) / \mathrm{N}}
$$

The interference in (19) can be further expressed as [27]

$$
\mu_{k}(m)=\sum_{n=0}^{N-1} \frac{\sin \left(\pi \varepsilon_{k^{\prime}}\right)}{N \sin \left(\pi\left(\varepsilon_{k^{\prime}}-\varepsilon_{k}\right) / N\right)} \cdot e^{j \pi\left(\varepsilon_{k^{\prime}}-\varepsilon_{k}\right)(N-1) / N} .
$$

As shown in Fig. 3, the chip-by-chip detection is carried out involving the ESE function, and the DECs perform the decoding operation using the output of the ESE as the input. Now considering the scenario where detection takes place in a multipath channel, with BPSK signaling assumed, the interference mean and variance based on [29] are given by

$$
\begin{gathered}
E\left(\zeta_{k}^{\prime}(m)\right)=E\left(R_{c}(m)\right)-H_{k^{\prime}}(m) \mu_{k}(m) E\left(X_{k^{\prime}}(m)\right) \\
\operatorname{Var}\left(\zeta_{k}^{\prime}(m)\right)=\operatorname{Var}\left(R_{c}(m)\right)-\left|H_{k^{\prime}}(m) \mu_{k}(m)\right|^{2} \operatorname{Var}\left(X_{k^{\prime}}(m)\right)
\end{gathered}
$$

From the equations above, the output of the elementary signal operator in the presence of CFO, in a multipath channel, based on the extrinsic log-likelihood ratios (LLRs) generation [29], is represented by

$$
e_{E S E}^{\prime}\left(X_{k}(m)\right)=2 H_{k}(m) \frac{R_{c}(m)-E\left(\zeta_{k}^{\prime}(m)\right)}{\operatorname{Var}\left(\zeta_{k}^{\prime}(m)\right)}
$$

The above equations therefore represent the expressions for the OFDM-IDMA system model in the presence of carrier frequency offset. The model used combines the signal for all users at the receiver. The established presence of CFO and its degrading impact on the OFDM-IDMA system in a multipath channel should therefore necessitate the need for an effective correction and synchronization technique to improve the performance and the efficiency of the multiuser scheme. 


\section{The LMS-BASED Adaptive Synchronization Algorithm}

The carrier frequency offset of a particular user can be compensated coarsely in the time domain of the system. But the main challenge is the residual CFOs due to multiple active users in the system, which results in interchannel interference (ICI). Due to system configuration of the OFDM-IDMA scheme, the mitigation of the effect of the residual CFOs is executed after the fast Fourier transforms (FFT) process, in the frequency domain. The proposed LMS-based adaptive synchronization algorithm is utilized to compensate the residual CFOs due to simultaneous users in the system. The characteristics of the LMS algorithm have been broadly studied and the most striking feature of the LMS-based algorithm is its simplicity [30]. The coefficient vector of the adaptive algorithm is updated by the expression [31]

$$
L(n+1)=L(n)+2 \mu \times e(n) x_{k}(n),
$$

where $L(n)$ is the coefficient vector, $\mu$ is the step-size which regulates the convergence speed of the synchronization algorithm and $x_{k}(n)$ is the modulated input signal utilized in the implementation of the algorithm. The update error signal $e(n)$ is expressed as

$$
e(n)=r(n)-r_{c}(n),
$$

where $r(n)$ represents the desired signal and $r_{c}(n)$ is the received signal in the presence of CFOs. The error signal is therefore used to update the carrier frequency-tracking loop, and the expected output is represented as [31]

$$
y(n)=L(n) x_{k}(n),
$$

Thus, with adequate knowledge of the input data by the receiver and the update error signal obtained, the impact of the CFOs can successfully be corrected for the users in the OFDM-IDMA system.

The non-recursive form of (24) is expressed as

$$
L(n)=L(0)+2 \mu \sum_{i=0}^{N-1} e(i) x_{k}(n) .
$$

From (16) and (17), initializing $\mathrm{L}(0)=0, y(n)$ is derived as

$$
y(n)=2 \mu \sum_{i=0}^{N-1} e(i) x_{k}^{\prime}(n), x_{k}(n)
$$

It is essential however, to state that the derived synchronization method is sensitive to the step-size as it is the case for most adaptive algorithms. Hence, 
the step-size $\mu$ is carefully determined [32] to obtain an efficient result and a reasonable convergence time.

\section{Simulation Results and Discussion}

This section presents the computer simulations carried out to analyze and substantiate the performance of the OFDM-IDMA system with CFO in fading multipath channel scenario. The OFDM-IDMA system model used has four users for all instances with input data length 32, spreading length 4 . The number of sub-carriers is $\mathrm{N}=128$, the number of samples in the guard interval is set at 7 and the number of iteration is 4 . In practice, the number of sub-carriers $\mathrm{N}$ is varied to provide variable data rates for different users. However, the same number of sub-carriers is assumed for all users in the simulation for convenience. The QPSK modulation technique is used assuming operating carrier frequency of $2 \mathrm{GHz}$ with sampling period of $0.5 \mu \mathrm{s}$. All simulation results are presented based on the bit error rate (BER) [33] performance of the system in a Rayleigh fading multipath channel of $\mathrm{M}=16$ paths with normalized Doppler frequencies of $\mathrm{fDn}=0.0136, \mathrm{fDn}=0.1085$ and $\mathrm{fDn}=0.1808$ corresponding to mobile speeds of $\mathrm{v}=15 \mathrm{~km} / \mathrm{h}, \mathrm{v}=120 \mathrm{~km} / \mathrm{h}$ and $\mathrm{v}=200 \mathrm{~km} / \mathrm{h}$ respectively. The general performance of the system is investigated for a constant slow fading scenario with mobile speed $\mathrm{v}=15 \mathrm{~km} / \mathrm{h}$ and then at increasing mobile speeds $\mathrm{v}=120 \mathrm{~km} / \mathrm{h}$ and $\mathrm{v}=200 \mathrm{~km} / \mathrm{h}$.

In Fig. 4, the general performance of an OFDM-IDMA system in the presence of CFOs is shown. Various values of CFOs are varied from zero (when there is no synchronization error in the system) to $\mathrm{CFO}=0.18$, with the mobile speed constant at $15 \mathrm{~km} / \mathrm{h}$ in the fading Rayleigh multipath channel. It can be seen from the plot that at small value of CFO (i.e. 0.02), the system performance is not significantly affected. But as the values increase, performance degradation increases as well.

Figure 5 shows the impact of increasing mobile speed on the OFDM-IDMA system in a fast fading Rayleigh multipath channel. The performance of the system is affected as the mobile speed is increased. It can be seen that at $\mathrm{CFO}=0.1$, with the mobile speed also increasing in a fast fading Rayleigh channel scenario, as shown in figure 5, there is significant degradation in the system performance. This means further increase in the value of CFO in the fast fading multipath channel scenario, will only worsen the performance of the system. 


\section{Muyiwa B. Balogun, et al}

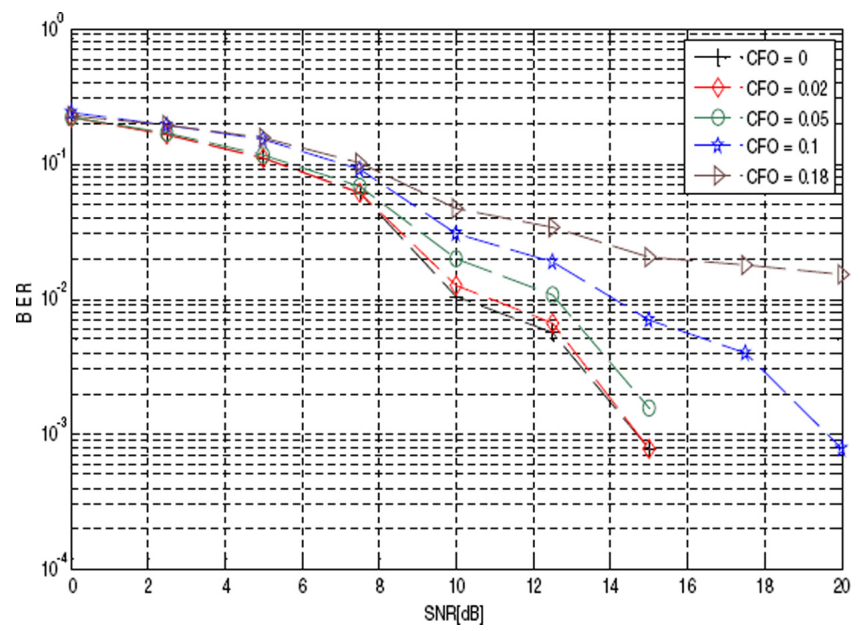

Figure 4 General BER performance of the OFDM-IDMA system model with increasing CFOs

In Fig. 6, the bit error rate performance of the OFDM-IDMA system with the application of the proposed LMS-based adaptive synchronization algorithm is presented. The plot shows the impact of the proposed algorithm on the system model in the presence of CFOs of 0.05 and 0.1 . There is an appreciable reduction and significant

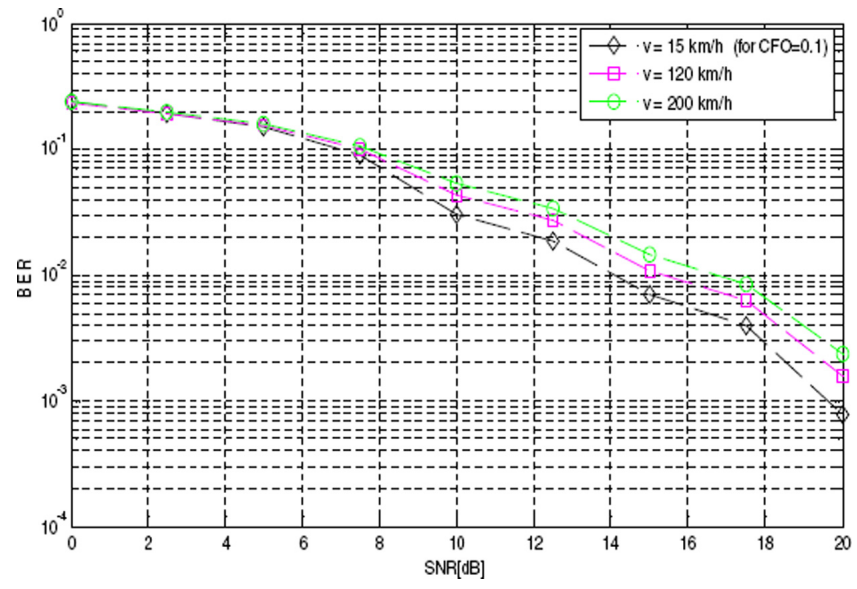

Figure 5 Performance result with increasing mobile speed at $\mathrm{CFO}=0.1$ 


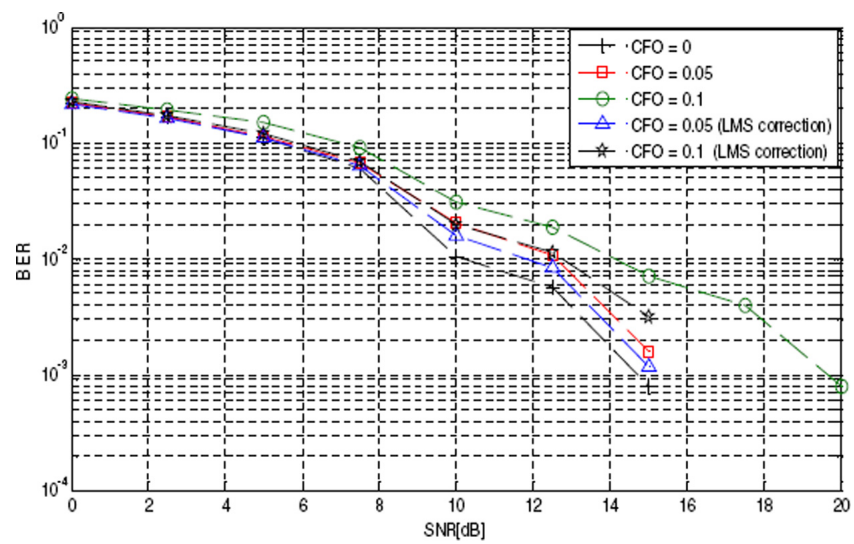

Figure 6 The impact of the proposed KLMS synchronization algorithm on the OFDM-IDMA system model with carrier frequency offsets 0.05 and 0.1

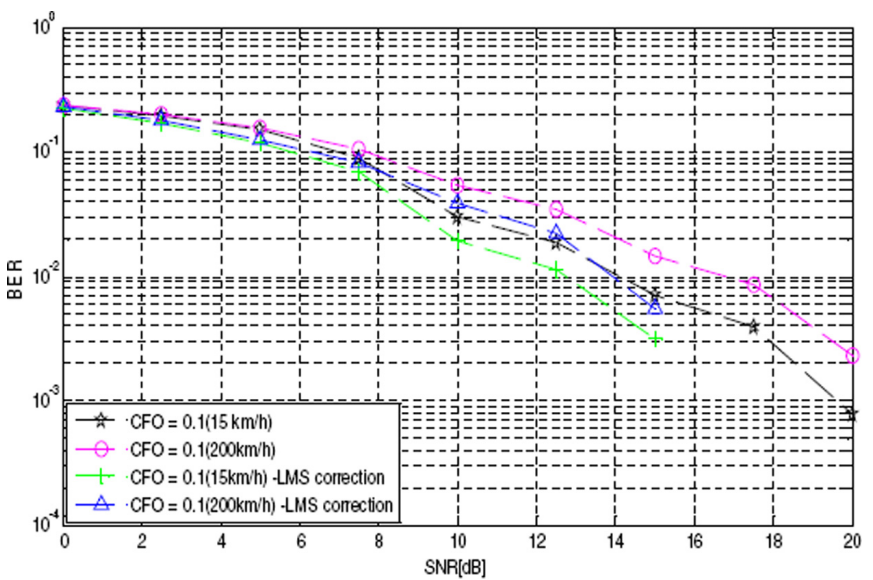

Figure 7 BER performance of the OFDM-IDMA system model with the proposed algorithm in both slow fading and fast fading multipath channel of mobile speeds $15 \mathrm{~km} / \mathrm{h}$ and $200 \mathrm{~km} / \mathrm{h}$ respectively

mitigation of the carrier frequency offset errors upon the application of the proposed algorithm. This signifies an effective reduction in the ICI due to the residual CFOs of concurrent users in the multicarrier IDMA system.

Furthermore, the performance of the OFDM-IDMA system model in a multipath channel of varying mobile speed is demonstrated in Fig. 7. The carrier frequency offset value is fixed while the mobile speed is varied at $15 \mathrm{~km} / \mathrm{h}$, and $200 \mathrm{~km} / \mathrm{h}$. The overall bit-error rate performance of the system 


\section{Muyiwa B. Balogun, et al}

degrades as the mobile speed increases. The introduction of the proposed algorithm, however, is able to mitigate the overall impact of carrier frequency offset error on the system, even in a fast fading multipath channel scenario.

\section{Conclusion}

The performance of the OFDM-IDMA system has been investigated and analyzed in a fast-fading Rayleigh multipath channel, in the presence of CFO. Simulation results clearly show that CFOs impact the system performance adversely in contrast to many research works on the OFDM-IDMA where it is assumed that CFOs have no influence on the overall performance of the system. Also, there is further degradation of the system in a fast fading multipath channel. An adaptive synchronization algorithm, which is LMS-based, has been presented to mitigate the impact of carrier frequency offset errors on the recently proposed OFDM-IDMA scheme. The algorithm focuses on the reduction of the ICI due to the residual CFOs from multiple active users in the system. The proposed algorithm was carried out in the presence of high carrier frequency offset values for clear demonstration of its efficiency. Simulation results also show an appreciable reduction and significant mitigation of the carrier frequency offset errors upon the application of the proposed algorithm in a fast fading Rayleigh multipath channel.

\section{Reference}

[1] R. L. Pickholtz, L. B. Milstein and D. L. Schilling. Spread spectrum for mobile communications, IEEE Trans. Vehicular Technology, vol. 40, pp. 313-322, May 1991.

[2] R. Prasad and S. Hara. An overview of multi-carrier CDMA, Int. Symp. IEEE Spread Spectrum Techniques and Applications Proceedings, vol. 1, pp. 107-114, Sept. 1996.

[3] A. McCormick, E. Al-Susa. Multicarrier CDMA for future generation mobile communications, IEE Electronics \& Comm.," Engineering, Vol. 14, Issue 2, Page(s): 52-60, April 2002.

[4] S. Moshavi. Multi-user Detection for DS-CDMACommunications. IEEE Commun. Mag., vol. 34, pp.124-36, Oct. 1996. 
[5] D. W. Matolak, V. Deepak, and F. A. Alder. Performance of Multitone and Multicarrier DS-SS in the presence of imperfect phase synchronization, MILCOM 2002, vol. 2, pp. 1002-1006, Oct 2002.

[6] K. Rasadurai and N. Kumaratharan. Performance enhancement of MCCDMA system through turbo multi-user detection, Computer comm. and Infomatics (ICCI) 2012, pp. 1-7, 2012.

[7] F. Corlier and F. Nouvel. Unsupervised neural network for Multi-user detection in MC-CDMA systems," IEEE Int. Conf. on Personal wireless comm., pp. 255-259, 2002.

[8] Li Ping, K. Y.Wu, L. H Liu and W. K. Leung, A simple unified approach to nearly optimal multiuser detection and space-time coding, Information TheoryWorkshop, ITW'2002, pp. 53-56, October 2002.

[9] Li Ping. Interleave-division multiple access and chip-by-chip iterative multi-user detection, IEEE Commun. Mag., vol. 43, no. 6, pp. S19-S23, June 2005.

[10] I. Mahafeno, C. Langlais, and C. Jego. OFDM-IDM Aversus IDMA with ISI cancellation for quasi-static Rayleigh fading multipath channels, in Proc. 4th Int. Symp. on Turbo Codes \& Related Topics, Munich, Germany, Apr. 3-7, 2006.

[11] K. Kusume, G. Bauch, W. Utschick. IDMA vs. CDMA: analysis and comparison of two multiple access schemes, IEEE Trans. Wireless Commun., vol. 11, pp. 78-87, Jan. 2012.

[12] Li Ping, Qinghua Guo and Jun Tong. The OFDM-IDMA Approach to Wireless Communication System, IEEE Wireless Communication, pp.18-24, June 2007.

[13] M. Morelli, A. D'Andrea, and U. Mengali, Feedback frequency synchronization for OFDM applications, IEEE Communication Letter, vol. 5, pp. 134-136, Jan. 2001.

[14] M. B Balogun, O. O. Oyerinde, and S. H. Mneney, "Performance Analysis of the OFDM-IDMA System with Carrier Frequency Offset in a Fast Fading Multipath Channel, in IEEE 3rd Wireless Vitae Conference, USA, June 24-27, 2013.

[15] L. Cimini. Analysis and Simulation of a Digital Mobile Channel Using Orthogonal Frequency Division Mutiplexing, IEEE Trans. Communication, vol. 33, no. 7, pp. 665-675, July 1985.

[16] A. Molisch. Orthogonal Frequency Division Multiplexing (OFDM), Wiley-IEEE Press eBook Chapters, second edition, pp. 417-43, 2011.

[17] Wireless LAN Medium Access Control (MAC) and Physical Layer (PHY) Specification: High-speed Physical Layer in the $5 \mathrm{GHz}$ Band, 
The Institute of Electrical and Electronics Engineers, Inc., IEEE Std. 802.1la-1999

[18] L. Liu,W. K. Leung, and Li Ping. Simple chip-by-chip multi-user detection for CDMAsystems," in Proc. IEEE VTC-Spring, Korea, pp. 2157-2161, Apr. 2003.

[19] K. Li, X. Wang, and L. Ping. Analysis and Optimization of InterleaveDivision Multiple-Access Communication Systems, IEEE Trans. on Wireless Communications 2007, vol. 65, 1973.

[20] Q. Huang, K.-K. Ko, P. Wang, L. Ping, S. Chan. Interleave-division multiple-access based broadband wireless networks, Information theory workshop, pp. 502-506, 2006.

[21] L. Ping, L. Liu, K.Wu, and W. Leung. On interleave-division MultipleAccess, in IEEE International Conference on Communications, vol. 5, pp. 2869-2873, June 2004.

[22] L. Ping, Q. Guo and J. Tong. The OFDM-IDMA Approach to Wireless Communication System, IEEE Wireless Communication, pp.18-24, June 2007.

[23] L. Ping, L. Liu, and W. Leung. A simple approach to near-optimal multiuser detection: interleave-division multiple-access," in Proc. IEEE Wireless Comm. Networking (WCNC 2003), vol. 1, pp. 391-396, March 2003.

[24] B. Dongming, Y. Xinying. A new approach for carrier frequency offset estimation in OFDM communication system, IEEE communication Tech. Proc., ICCT 2003, vol.2, pp. 1922-1925, 2003.

[25] M. Morelli, C. Kuo, and M. Pun. Synchronization techniques for orthogonal frequency division multiple access (OFDMA): a tutorial review, Proc. IEEE, vol. 95, no. 7, pp. 1394-1427, July 2007.

[26] A. Al-Dweik and R. Hamila, "A highly efficient blind carrier frequency offset estimator for wireless OFDM systems," Proc. of the IEEE Int. Conf. on Consumer Electronics (ICCE '06), pp. 375-376, CA, USA, Jan. 2006.

[27] T. Peng, Y. Xiao, X. He and S. Li. Improved Detection of Uplink OFDM-IDMA Signals with Carrier Frequency Offsets, IEEE communication letter, vol. 165, pp. 646-649, May 2012.

[28] Y. Liu, X. Xiong, Z. Luo. Effect of Carrier Frequency Offsets on OFDM-IDMA Systems, 2012 2nd International Conference, pp. 209-302, 2012. 
[29] L. Ping, L. Liu, K. Y. Wu, and W. K. Leung. Interleaved-Division Multiple-Access, IEEE Trans. Wireless Communication, vol.4, pp. 938-947, April 2006.

[30] T. Shan and T. Kailath. Adaptive algorithms with an automatic gain control feature," IEEE Transactions on circuits and systems, vol. 35, no. 1, pp. 122-127, January 1988.

[31] H. Modaghegh, R. H Khosravi, S. A Manesh and H. S Yazdi. A new modeling algorithm-Normalized Kernel Least Mean Square, IEEE International conference on Innovations in Information technology, IIT 2009, pp. 120-124, 2009.

[32] E. Alameda-Hernandez, D. Blanco, D. P Ruiz and M. C Carrion. The Averaged, Overdetermined, and Generalized LMS Algorithm, IEEE Transactions on signal processing, vol. 55, no 12, pp. 5593-5603, December 2007.

[33] Y. Rahmatallah, N. Bouaynaya and S. Mohan. Bit Error Rate Performance of Linear Companding Transforms for PAPR Reduction in OFDM Systems, in IEEE Global Communications Conference (GLOBECOM 2011), Houston, Texas, December 2011.

\section{Biographies}

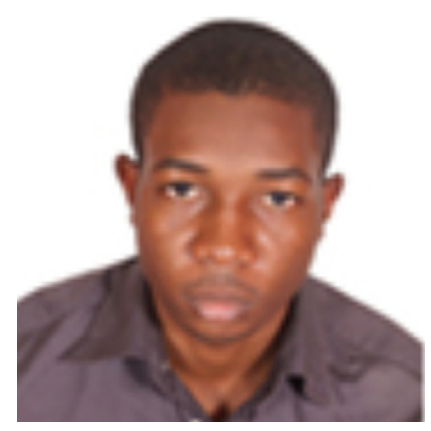

Muyiwa Blessing Balogun received the B.Sc (Hons) in 2009 from the University of Ilorin, Nigeria. He is presently studying towards his M.Sc degree at the University of Kwazulu-Natal, Durban, South Africa. His research interests include frequency synchronization algorithms for multicarrier systems, multiple antenna systems and digital signal processing applications. 


\section{Muyiwa B. Balogun, et al}

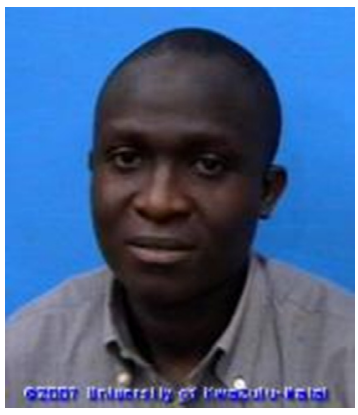

Olutayo Oyeyemi Oyerinde received the B.Sc. (Hons.) and the M.Sc. degrees in electrical and electronic engineering from Obafemi Awolowo University, Ile-Ife, Nigeria, in 2000 and 2004, respectively, and the Ph.D. degree in electronic engineering from the School of Engineering, University of KwaZulu-Natal (UKZN), Durban, South Africa, in 2010. He was a Postdoctoral Research Fellow with the School of Engineering, UKZN, under UKZN Postdoctoral Research Funding. He is currently a Telecommunications lecturer in the School of Electrical and Information Engineering, University of the Witwatersrand, South Africa. His research interests are in the area of wireless communications including multiple antenna systems, orthogonal frequency division multiplexing system and channels estimation, and signal processing techniques.

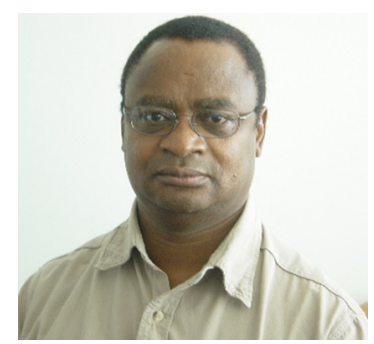

Stanley Henry Mneney received the B.Sc. (Hons.) Eng. degree from the University of Science and Technology, Kumasi, Ghana, in 1976 and the M.A.Sc. from the University of Toronto, Toronto, Ontario, Canada, in 1979. In a Nuffic funded project by the Netherlands government he embarked on a sandwich Ph.D programme between the Eindhoven University of Technology, Eindhoven, Netherlands and the University of Dares Salaam, Dares Salaam, Tanzania, the latter awarding the degree in 1988. He is at present a Professor of Telecommunication and Signal Processing and head of the Radio Access 
and Rural Telecommunication (RART) Centre in the School of Engineering, University of KwaZulu-Natal, Durban, South Africa. His research interests include theory and performance of telecommunication systems, low cost rural telecommunications services and networks, channel modelling and digital signal processing applications. 
